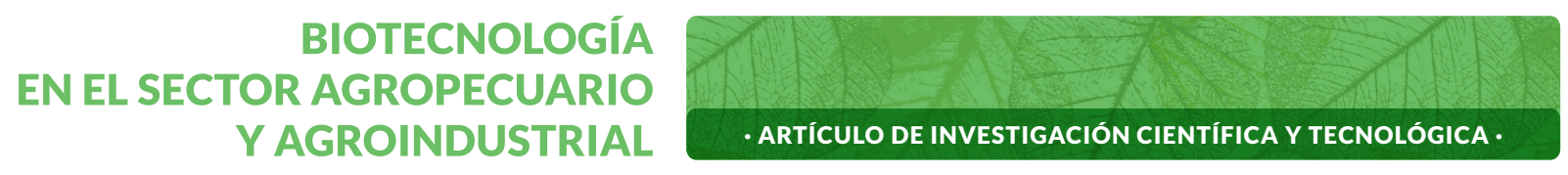

\title{
Efecto de Bacillus subtilis sobre metabolitos sanguíneos y parámetros productivos en pollo de engorde*
}

\author{
Effect of Bacillus subtilis on blood \\ metabolites and productive parameters \\ in broiler chickens
}

\section{Efeito de Bacillus subtilis sobre metabólitos sanguíneos e parâmetros produtivos em frangos de corte}

\author{
MAYA-ORTEGA, CARLOS-ABEL ${ }^{1}$; MADRID-GARCÉS, TOMÁS-ANTONIO²; \\ PARRA-SUESCÚN, JAIME-EDUARDO ${ }^{3}$
}

Historial del Artículo

Recibido para evaluación: 23 de Abril 2020.

Aprobado para publicación: 20 de Octubre 2020.

* Título del proyecto de origen: "Evaluación de Bacillus subtilis sobre el desarrollo alométrico de órganos digestivos y variables productivas en pollo de engorde". Entidad financiadora: Universidad Nacional de Colombia, Convocatoria:registro único de proyectos, código HERMES 37384. Finalización: 5 de Diciembre de 2019.

1 Universidad Nacional de Colombia, Sede Medellín, Facultad de Ciencias Agrarias, Departamento de Producción Animal, Grupo de investigación BIOGEM. Zootecnista Msc. Medellín, Colombia. https://orcid.org/0000-0002-5094-7716

2 Universidad Nacional de Colombia, Sede Medellín, Facultad de Ciencias Agrarias, Departamento de Producción Animal, Grupo de investigación BIOGEM. Zootecnista MsC, Ph.D(c) Ciencias agrarias. Medellín, Colombia. https://orcid.org/0000-0002-4191-2343

3 Universidad Nacional de Colombia, Sede Medellín, Facultad de Ciencias Agrarias, Departamento de Producción Animal, Grupo de investigación BIOGEM. Zootecnista MsC., Ph.D. Ciencias agrarias. Medellín, Colombia. https://orcid.org/0000-0003-4772-1326

Correspondencia: camayao@unal.edu.co 


\section{RESUMEN}

Los antibióticos han sido utilizados en la alimentación animal con el fin de garantizar una producción más eficiente; sin embargo, la aparición de bacterias resistentes a los antibióticos ha causado preocupación tanto a consumidores como a científicos. El objetivo fue evaluar el efecto de Bacillus subtilis sobre parámetros sanguíneos y productivos en pollos de engorde. 72 pollos de la línea Cobb 500, fueron aleatorizados a 3 dietas: dieta basal sin antibioticos (D1), y una dieta basal suplementada con 10 ppm de Avilamicina (D2), o 50 ppm de esporas de B. subtilis (D3) durante 42 días. Los días 21 y 42 se estimó la ganancia acumulada de peso (GAP), la conversión alimenticia (CA) y se evaluaron las concentraciones sanguíneas de alanina aminotransferasa (ALT), creatinina, triglicéridos, colesterol, glucosa, calcio y fósforo. El uso B. subtilis en la dieta mejoró el rendimiento productivo de las aves, disminuyó las concentraciones séricas de triglicéridos, colesterol y ALT, e incrementó las concentraciones de calcio, fosforo y glucosa. La inclusión de B. subtilis en la dieta de pollos de engorde es una alternativa al uso de antibióticos, ya que tiene efectos favorables sobre la bioquímica sanguínea y el rendimiento productivo de las aves.

\section{ABSTRACT}

Antibiotics have been used in animal feed in order to ensure more efficient production, however, the appearance of antibiotic resistant bacteria has caused concern in both consumers and scientific. The aim was to evaluate the effect of Bacillus subtilis on blood parameters and performance in broilers. $72 \mathrm{Cobb}$ 500 broiler chicks, were randomized to 3 diets. A basal diet without antibiotics (D1), or a basal diet supplemented with either 10 ppm of avilamicyn (D2) or 50 ppm of spores of B. subtilis (D3) during 42 days. On days 21 and 42 cumulative weight gain (BW), feed conversion (FCR) was estimated and blood concentrations of alanine aminotransferase (ALT), creatinine, triglycerides, cholesterol, glucose, calcium and phosphorus were evaluated. The use of $B$. subtilis in the diet improves the performance of broilers, lowers the blood levels of triglycerides, cholesterol and ALT blood concentrations, and increase the blood levels of calcium, phosphorus and glucose. The inclusion of B. subtilis in the diet of broilers is an alternative to the use of antibiotics, as it has positive effects on the blood biochemistry and the performance.

\section{RESUMO}

Antibióticos têm sido utilizados na alimentação animal, a fim de garantir uma produção mais eficiente; no entanto, o surgimento de bactérias resistentes a antibióticos causou preocupação a consumidores e cientistas. $O$ objetivo foi

Cómo citar este artículo: MAYA-ORTEGA, CARLOS-ABEL; MADRID-GARCÉS, TOMÁS-ANTONIO; PARRA-SUESCÚN, JAIME-EDUARDO. Efecto de Bacillus subtilis sobre metabolitos sanguíneos y parámetros productivos en pollo de engorde. Biotecnología en el sector agropecuario y agroindustrial, v. 19, n. 1, 2021, p. 105-116. Doi: https://doi.org/10.18684/BSAA(19)105-116

\section{PALABRAS CLAVE:}

Antibiótico; Avicultura; Bioquímica Sanguínea; Probiótico; Rendimiento Productivo.

\section{KEYWORDS:}

Antibiotic; Poultry; Blood biochemistry; Probiotic; Performance.

\section{PALAVRAS-CHAVE:}

Antibiótico; Avicultura; Bioquímica do sangue; Probiótico; Rendimento produtivo. 
avaliar o efeito de Bacillus subtilis no sangue e parâmetros produtivos em frangos de corte. 72 galinhas da linha Cobb 500 foram randomizadas para 3 dietas. Uma dieta basal sem antibióticos (D1) ou uma dieta basal suplementada com 10 ppm de Avilamicina (D2) ou 50 ppm de esporos de B. subtilis (D3) por 42 dias. Nos dias 21 e 42, foi estimado o ganho de peso acumulado (GAP), a conversão alimentar (CA) e os níveis sanguíneos de alanina aminotransferase (ALT), creatinina, triglicerídeos, colesterol, glicose, cálcio e fósforo. O uso de B. subtilis na dieta melhorou o desempenho produtivo das aves, diminuiu as concentrações séricas de triglicerídeos, colesterol e ALT e aumentou as concentrações de cálcio, fósforo e glicose. A inclusão de B. subtilis na dieta de frangos de corte é uma alternativa ao uso de antibióticos, pois possui efeitos favoráveis na bioquímica sanguínea e no desempenho produtivo de aves.

\section{INTRODUCCIÓN}

El incremento en la demanda de alimentos como consecuencia del crecimiento acelerado de la población mundial, ha llevado a los sistemas pecuarios a ser cada vez más eficientes, con el fin de producir alimentos en grandes volúmenes que cumplan con los estándares sanitarios y las exigencias del consumidor [1].

Con el fin de satisfacer las demandas de proteína de origen animal, la industria avícola evolucionó a producciones cada vez más intensivas. Según reportes de la FAO, se tiene proyectado que, en el año 2020, la carne de pollo será la más consumida a nivel mundial [2]. Actualmente, se cuenta con aves genéticamente más productivas y eficientes, pero menos adaptadas a condiciones de estrés y más susceptibles a problemas de salud y desequilibrios en la microbiota normal del intestino [2,3].

Durante los últimos años, los antibióticos han sido utilizados en la alimentación avícola como una medida para garantizar altos niveles de producción, eficiencia y rentabilidad de las granjas, además de brindar mejor salud y bienestar a las aves [4,5]. Sin embargo, la exposición de los animales a dosis subterapéuticas diarias de antibióticos como una medida profiláctica independiente de su estado de salud, ha conllevado a la aparición de cepas bacterianas resistentes a antibióticos, a la acumulación de estos residuos en el producto final, y a la presentación de desbalances en la microbiota normal del intestino [5]. Debido a lo anterior, y a la creciente preocupación de la comunidad científica, la unión europea prohibió el uso de antibióticos como promotores de crecimiento (APC) en la producción animal desde enero de 2006 [3,6].

Dado que la prohibición de los APC provoca un impacto desfavorable sobre la producción, los investigadores se han dado a la tarea de buscar alternativas biológicamente seguras para el consumidor final, entre las que se ha estudiado el uso de probióticos [7,8,9]. Los probióticos son definidos como microorganismos vivos no patógenos, que cuando son administrados en cantidades adecuadas ejercen efectos benéficos sobre la salud del hospedador [7,10]. Entre la innumerable cantidad de probióticos que se han estudiado, Bacillus subtilis ha presentado atributos que permiten su utilización en la alimentación animal, tales como la capacidad de generar esporas bajo situaciones de estrés, lo que le permite tolerar altas temperaturas durante la elaboración del alimento balanceado, y resistir el pH ácido del estómago; además, Bacillus subtilis ejerce efectos benéficos a nivel intestinal como la estimulación del sistema inmune, el desarrollo del tracto gastrointestinal y la modulación/cambio de la microbiota intestinal [7,11,12].

Los cambios en la composición microbiana, debido a bacterias indeseadas (patógenas) en intestino, puede ser causada por diferentes factores, principalmente alimenticios; estos desbalances microbianos pueden afectar notablemente el metabolismo y verse reflejado a nivel sanguíneo por diferentes compuestos e intermediarios del metabolismo de aminoácidos, lípidos y carbohidratos [13]. El estudio y análisis de algunos parámetros bioquímicos sanguíneos permiten hacer un monitoreo de la integridad del tracto gastrointestinal, funcionalidad de órganos indispensables en los procesos digestivos como el hígado y el páncreas, identificar enfermedades metabólicas y deficiencias nutricionales sin afectar el bienestar y comportamiento productivo del animal, por se evaluó el efecto de Bacillus subtilis como alternativa al uso de antibioricos promotores de crecimietno sobre parametros sanguineos como alanina aminotransferasa, creatinina, fósforo, calcio, glucosa, triglicéridos y colesterol y su relación con parametros productivos en pollos de engorde. 


\section{MÉTODO}

\section{Consideraciones éticas}

Los procedimientos experimentales llevados a cabo dentro del estudio siguieron los lineamientos estipulados en las guías "The International Guiding Principles for Biomedical Research Involving Animals" (2012). Esta investigación fue avalada por El Comité Institucional para el Cuidado y Uso de los Animales-CICUA de la Universidad Nacional de Colombia, Sede Medellín (CEMED-013. Mayo 04 de 2018).

\section{Localización}

El trabajo de campo se realizó en las instalaciones de la Estación Agraria San Pablo, perteneciente a la Universidad Nacional de Colombia (Sede Medellín), ubicada en el municipio de Rionegro (oriente de Antioquia), vereda El Tablazo, a una altitud promedio de $2.100 \mathrm{msnm}$, con una temperatura entre 12 y $18^{\circ} \mathrm{C}$ y humedad relativa del $75 \%$, en una zona de vida bosque muy húmedo montano bajo (bmh-MB).

\section{Animales}

Para la realización del estudio se utilizaron 72 animales de la línea genética ROSS308, de un día de nacidos, suministrados por una casa genética comercial. Los animales se alojaron en corrales de piso de 0,25 $\mathrm{m}^{2}$ para recrear las condiciones de densidad de una producción comercial y las prácticas de manejo, plan vacunal (día 8 y 20 de vida se aplicó Gumboro y Newcastle vía ocular) y equipos empleados fueron similares a los implementados en una granja comercial. El periodo experimental tuvo una duración de 42 días.

\section{Manejo sanitario}

El galpón donde se recibieron las aves se preparó previamente teniendo en cuenta todos los protocolos de bioseguridad: lavado, limpieza y desinfección de las instalaciones, cortinas, criadoras, comederos y bebederos. Adicionalmente, se realizó un estricto control de roedores e insectos. Cinco horas antes de la llegada de los pollitos, las criadoras fueron encendidas, con la finalidad de precalentar el galpón y alcanzar una temperatura promedio de $32^{\circ} \mathrm{C}$ al momento de la recepción de los mismos [14].

\section{Dietas}

Las aves fueron alimentadas con una dieta basal (cuadro 1) que consistió en una dieta comercial sin la adición de antibiótico o probiótico (Bacillus subtilis), conformando cada una de las dietas así: dieta basal (D1): sin la adición de antibiótico y probiótico (Bacillus subtilis), dieta 2 (D2): dieta basal más la adición de antibiótico promotor de crecimiento (APC-avilamicina) y dieta 3 (D3): dieta basal más la adición de probiótico (Bacillus subtilis).

El plan de alimentación fue dividido en dos etapas, para lo cual se realizó una dieta de iniciación (1-21 días) y finalización (22-42 días), cumpliendo los requerimientos nutricionales establecidos por la distribuidora comercial. La adición del antibiótico (avilamicina) en el alimento (D2), se realizó según las indicaciones de la casa productora, a razón de 10 ppm. La cantidad de Bacillus subtilis incorporada en D3, se llevó a cabo atendiendo la recomendación del fabricante, esto es 50 ppm para garantizar una dosis de $10^{8}$ UFC.

\section{Cuantificación de metabolitos sanguíneos}

Para este análisis se tomaron $5 \mathrm{~mL}$ de sangre por ave los días 1, 21 y 42 . Haciendo uso de una aguja de $25 \mathrm{~mm}$ se realizó punción cardiaca, específicamente en el ventrículo izquierdo, para la extracción de sangre en un tubo de ensayo con anticoagulante. Cada una de las muestras se conservó en frío a $4^{\circ} \mathrm{C}$ para su transporte al laboratorio, donde fueron centrifugadas a 1600 rpm durante 10 minutos para separar el plasma en alícuotas 
y posteriormente ser analizadas [13]. Se realizaron mediciones de los siguientes metabolitos: Alanina aminotransferasa-ALT (U/L), creatinina (mg/dL), fósforo (mg/dL), calcio ( $\mathrm{mg} / \mathrm{dL})$, glucosa (mg/dL), triglicéridos (mg/dL) y colesterol (mg/dL). La cuantificación en suero se realizó mediante un estuche comercial (DIA.PRO Diagnostic Bioprobes), siguiendo las instrucciones del fabricante.

\section{Determinación de parámetros productivos}

Los días 21 y 42 del experimento, se evaluaron los siguientes parámetros productivos (Ecuaciones 1 y 2) [15,16]:

Conversión alimenticia acumulada (CA)

$$
C A=\frac{\text { Alimento consumido }}{\text { Peso ganado }}
$$

Ganancia de peso acumulada (GAP):

$$
G A P=\frac{\text { Peso final }- \text { Peso inicial }}{\text { Edad }(\text { días })}
$$

Cuadro 1. Aporte nutricional de la dieta basal (D1) diseñada en dos etapas (iniciación y finalización).

\begin{tabular}{|l|c|c|c|}
\hline \multicolumn{2}{|c|}{ Aporte nutricional por etapa } & Iniciación & Finalización \\
\hline Hutrientes & Unidad & Valor & Valor \\
\hline E.m. aves & $\%$ & 10,926 & 10,845 \\
\hline Proteina bruta & KCAL/kg & 3152 & 3299 \\
\hline Grasa & $\%$ & 21,474 & 19,976 \\
\hline Extracto Libre de N (ELN) & $\%$ & 8,301 & 10,213 \\
\hline Fibra bruta & $\%$ & 49,673 & 50,195 \\
\hline Cenizas & $\%$ & 2,927 & 2,801 \\
\hline Calcio & $\%$ & 6,108 & 5,379 \\
\hline Fósforo disp. & $\%$ & 0,997 & 0,832 \\
\hline Fósforo total & $\%$ & 0,418 & 0,360 \\
\hline B. Electrolítico & $\%$ & 0,648 & 0,580 \\
\hline Lisina & $\%$ Eqkg & 216,164 & 191,553 \\
\hline Metionina & $\%$ & 1,363 & 1270 \\
\hline Met + cist & $\%$ & 0,650 & 0,602 \\
\hline Treonina & $\%$ & 0,993 & 0,924 \\
\hline Triptófano & $\%$ & 0,901 & 0,825 \\
\hline Arginina & $\%$ & 0,242 & 0,222 \\
\hline Isoleucina & $\%$ & 1,336 & 1,228 \\
\hline Leucina & $\%$ & 0,881 & 0,815 \\
\hline Valina & $\%$ & 1,902 & 1,802 \\
\hline Histidina & $\%$ & 1,042 & 0,915 \\
\hline Fenilalanina & $\%, 547$ & 0,509 \\
\hline Fenil \& tiro & $\%$ & 1,061 & 0,989 \\
\hline Glicina & $\%, 953$ & 1.103 \\
\hline Alanina & $\%, 875$ & \\
\hline
\end{tabular}




\section{Diseño estadístico}

Se realizó un modelo de parcelas divididas bajo un esquema de aleatorización completamente al azar con 6 tratamientos (dieta*edad) y 3 repeticiones (corral) por tratamiento (4 pollos por repetición). Las parcelas grandes estuvieron constituidas por las dietas y las subparcelas por los diferentes días de muestreo. Cada animal fue asignado aleatoriamente a cada uno de los tratamientos (dieta*edad). El análisis estadístico fue realizado haciendo uso del procedimiento GLM (Modelos Lineales Generales) PROC MIXED del SAS ${ }^{\circledR}$ software, versión 9.4 [17]. Se realizó una prueba de comparaciones multiples de medias mediante el método de diferenicia significativa honesta (DSH) de tuckey haciendo uso de la función MEANS TRAT del SAS ${ }$ software, versión 9.4 con el fin de determinar diferencias significativas.

\section{RESULTADOS}

En general, las aves presentaron un buen estado de salud, sin la presencia de signos adversos de enfermedad que causara su retiro y/o sacrificio antes de terminar el periodo experimental. Adicionalmente, las aves consumieron la ración diaria de alimento ajustada a la guía de manejo de la línea genética. En el análisis estadístico de los resultados no se encontró interacción estadística entre la dieta y la edad de muestreo para ninguna de las variables en estudio, por ello no se hizo necesario analizar cada uno de los factores de forma independiente.

En el cuadro 2 se presentan las concentraciones sanguíneas de alanina aminotransferasa y creatinina. La evaluación de alanina aminotransferasa (ALT) presentó diferencias estadísticamente significativas $(p<0,05)$ entre las dietas evaluadas al día 21 de edad, siendo el valor más bajo el de aquellas aves alimentadas con D3 (B. subtilis). En el día 42 de vida se observó diferencia significativa $(p<0,05)$ entre D1 y D3, donde D3 presentó los valores más bajos. De igual manera, los niveles de Creatinina presentaron diferencias estadísticamente significativas $(p<0,05)$ entre las dietas evaluadas, donde D1 presentó los valores más altos, mientras que D2 y D3 no mostraron diferencias significativas $(p>0,05)$ en ninguno de los periodos evaluados.

Los niveles de glucosa (cuadro 3) presentaron diferencias estadísticamente significativas $(p<0,05)$ entre las distintas dietas para los periodos evaluados, donde el uso de Bacillus subtilis en la dieta aumentó los niveles de glucosa a los 21 y 42 días con respecto a los demás tratamientos, mientras que D1 presentó los valores más bajos de glucosa.

Cuadro 2. Efecto de la dieta sobre las concentraciones sanguíneas de Alanina aminotransferasa y Creatinina analizadas a los 1, 21 y 42 días de edad.

\begin{tabular}{|c|c|c|c|c|}
\hline \multirow[t]{2}{*}{ Parámetros } & \multicolumn{3}{|c|}{ Edad } & \multirow[b]{2}{*}{ EEM } \\
\hline & 1 & 21 & 42 & \\
\hline \multicolumn{5}{|c|}{ Alanina aminotransferasa (UI/L) } \\
\hline D1 & $3,11^{\mathrm{A}}$ & $3,82^{A}$ & $8,50^{\mathrm{A}}$ & \multirow{3}{*}{0,60} \\
\hline D2 & $3,11^{\mathrm{A}}$ & $2,56^{\mathrm{B}}$ & $6,70^{A B}$ & \\
\hline D3 & $3,11^{\mathrm{A}}$ & $1,76^{c}$ & $5,65^{\mathrm{B}}$ & \\
\hline \multicolumn{5}{|c|}{ Creatinina (mg/dL) } \\
\hline D1 & $0,235^{\mathrm{A}}$ & $0,373^{A}$ & $0,274^{A}$ & \multirow{3}{*}{0,02} \\
\hline D2 & $0,235^{\mathrm{A}}$ & $0,325^{\mathrm{B}}$ & $0,255^{B}$ & \\
\hline D3 & $0,235^{\mathrm{A}}$ & $0,325^{B}$ & $0,250^{B}$ & \\
\hline \multicolumn{5}{|c|}{$\begin{array}{l}\text { A, B, C Comparaciones dentro de una misma columna, medias con diferente letra son } \\
\text { estadísticamente diferentes } \quad(P<0 ; 05) \text {. EEM: Error estándar de la media. D1: Dieta control } \\
\text { sin antibiótico ni probiótico. D2: Alimento comercial con antibiótico. D3: Alimento comercial } \\
\text { con Bacillus subtilis. }\end{array}$} \\
\hline
\end{tabular}


En el cuadro 3 se presentan las concentraciones sanguíneas de colesterol y triglicéridos. En cuanto a los niveles sanguíneos de colesterol, se presentaron diferencias estadísticamente significativas $(p<0,05)$ al día 21 entre las tres dietas estudiadas, mientras que al día 42 , no se presentaron diferencias significativas $(p>0,05)$ entre D2 y D3. Por su parte, los niveles de triglicéridos mostraron diferencias estadísticamente significativas $(p<0,05)$ entre las distintas dietas evaluadas. Los niveles más bajos de triglicéridos para los días 21 y 42 de vida fueron encontrados en aquellas aves que recibieron Bacillus subtilis en la dieta.

Cuadro 3. Efecto de la dieta sobre las concentraciones sanguíneas de glucosa, triglicéridos y colesterol analizados a los 1, 21 y 42 días de edad.

\begin{tabular}{|c|c|c|c|c|}
\hline \multirow[t]{2}{*}{ Parámetro } & \multicolumn{3}{|c|}{ Edad } & \multirow[b]{2}{*}{ EEM } \\
\hline & 1 & 21 & 42 & \\
\hline \multicolumn{5}{|c|}{ Triglicéridos (mg/dL) } \\
\hline D1 & $128,1^{\mathrm{A}}$ & $199,3^{\mathrm{A}}$ & $141,5^{\mathrm{A}}$ & \multirow{3}{*}{0,5} \\
\hline D2 & $128,1^{\mathrm{A}}$ & $194,1^{\mathrm{B}}$ & $134,0^{B}$ & \\
\hline D3 & $128,1^{\mathrm{A}}$ & $191,0^{c}$ & $127,0^{c}$ & \\
\hline \multicolumn{5}{|c|}{ Colesterol (mg/dL) } \\
\hline D1 & $131,7^{\mathrm{A}}$ & $186,0^{\mathrm{A}}$ & $144,5^{\mathrm{A}}$ & \multirow{3}{*}{0,4} \\
\hline D2 & $131,7^{A}$ & $182,5^{\mathrm{B}}$ & $136,5^{\mathrm{B}}$ & \\
\hline D3 & $131,8^{A}$ & $163,0^{c}$ & $134,0^{B}$ & \\
\hline \multicolumn{5}{|c|}{ Glucosa (mg/dL) } \\
\hline D1 & $267,3^{\mathrm{A}}$ & $287,3^{A}$ & $321,5^{\mathrm{A}}$ & \multirow{3}{*}{0,5} \\
\hline D2 & $267,3^{A}$ & $292,8^{B}$ & $337,5^{\mathrm{B}}$ & \\
\hline D3 & $267,3^{A}$ & $300,0^{c}$ & $351,0^{c}$ & \\
\hline \multicolumn{5}{|c|}{$\begin{array}{l}\text { A, B, C Comparaciones dentro de una misma columna, medias con diferente letra son } \\
\text { estadísticamente diferentes (P < 0,05). EEM: Error estándar de la media. D1: } \\
\text { Dieta control sin antibiótico ni probiótico. D2: Alimento comercial con antibiótico. } \\
\text { D3: Alimento comercial con Bacillus subtilis. }\end{array}$} \\
\hline
\end{tabular}

Finalmente, los valores de calcio y fósforo (cuadro 4) mostraron diferencias estadísticamente significativas $(p<0,05)$ entre las diferentes dietas, donde el uso de Bacillus subtilis en la dieta incrementó de manera significativa la concentración sanguínea de ambos nutrientes en sangre.

Cuadro 4. Efecto de la dieta sobre las concentraciones sanguíneas de calcio y fósforo analizados a los 1, 21 y 42 días de edad.

\begin{tabular}{|c|c|c|c|c|}
\hline \multirow[t]{2}{*}{ Parámetros } & \multicolumn{3}{|c|}{ Edad } & \multirow[b]{2}{*}{ EEM } \\
\hline & 1 & 21 & 42 & \\
\hline \multicolumn{5}{|c|}{ Calcio (mg/dL) } \\
\hline D1 & $8,890^{A}$ & $10,52^{A}$ & $11,51^{\mathrm{A}}$ & \multirow{3}{*}{0,02} \\
\hline D2 & $8,890^{A}$ & $10,74^{B}$ & $11,74^{\mathrm{B}}$ & \\
\hline D3 & $8,890^{A}$ & $11,53^{c}$ & $13,09^{c}$ & \\
\hline \multicolumn{5}{|c|}{ Fóforo (mg/dL) } \\
\hline D1 & $7,100^{A}$ & $8,160^{A}$ & $8,945^{A}$ & \multirow{3}{*}{0,03} \\
\hline D2 & $7,100^{A}$ & $8,850^{B}$ & $9,631^{B}$ & \\
\hline D3 & $7,100^{A}$ & $9,155^{c}$ & $10,35^{c}$ & \\
\hline \multicolumn{5}{|c|}{$\begin{array}{l}\text { A,B,C Comparaciones dentro de una misma columna, medias con diferente letra son } \\
\text { estadísticamente diferentes } \quad(P<0 ; 05) \text {. EEM: Error estándar de la media. D1: } \\
\text { Dieta control sin antibiótico ni probiótico. D2: Alimento comercial con antibiótico. } \\
\text { D3: Alimento comercial con Bacillus subtilis. }\end{array}$} \\
\hline
\end{tabular}


En el cuadro 4 se presentan los resultados obtenidos para las variables productivas evaluadas. En cuanto a la tasa de conversión alimenticia no se presentó diferencia estadísticamente significativa $(p>0,05)$ entre las diferentes dietas al día 21; no obstante, dicho parámetro presentó diferencias estadísticamente significativas $(p<0 ; 05)$ entre dietas el día 42, donde la adición de Bacillus subtilis evidencia un efecto similar al uso de avilamicina como APC. Por su parte, la ganancia acumulada de peso fue significativamente más alta en las aves que recibieron el probiótico en comparación con las demás dietas.

Estudios previos han demostrado una disminución de los niveles sanguíneos de ALT en aves que fueron alimentados con dietas que contenían Bacillus subtilis, Bacillus coagulans y Saccharomyces boulardii $[18,19,20]$. Abramowicz et al., 2019, encontraron que el uso de cultivos vivos de B. subtilis en la dieta reduce de manera significativa la actividad de la alanina transferasa.

Niveles bajos de ALT están asociados a una buena salud y funcionalidad del hígado, y a la ausencia de agentes patógenos, como E. coli, en el organismo [7,20,21,22].

Bioensayos llevados a cabo por Sharma et al., (2015) en pollos que recibieron una mezcla de probióticos del género Bacillus, presentaron concentraciones bajas de creatinina en sangre, en comparación con aquellas aves que fueron adicionadas con Bacitracina de zinc en el alimento [22]. De manera similar, en el presente estudio las aves que recibieron Bacillus subtilis en la dieta presentaron valores más bajos de creatinina en sangre. Estudios en los cuales se han evaluado los niveles sanguíneos de creatinina, han correlacionado niveles altos de creatinina en pollos infectados con el virus de Newcastle [23].

Se ha reportado que algunos microorganismos probióticos tienen la habilidad de modificar las concentraciones de colesterol en sangre, a través de la disgregación de sales biliares en compuestos biliares libres o ligando directamente el colesterol proveniente de la dieta durante el proceso de digestión de las grasas, disminuyendo así la absorción de colesterol en el tracto gastrointestinal de las aves [24,25]. Al analizar los resultados obtenidos en el presente estudio, los niveles de colesterol y triglicéridos en sangre, fueron menores en aquellas aves que recibieron Bacillus subtilis en la dieta; estudios previos en los cuales se ha utilizado Bacillus subtilis como probiótico han mostrado resultados similares a los de este estudio [18,20,25,26,27].

Cuadro 5. Parámetros productivos evaluados los días 21 y 42 en pollos alimentados con una dieta basal o dieta basal suplementada con antibiótico (avilamicina) o probiótico (Bacillus subtilis).

\begin{tabular}{|c|c|c|c|}
\hline \multirow[t]{2}{*}{ Parámetros } & \multicolumn{2}{|c|}{ Edad } & \multirow[b]{2}{*}{ EEM } \\
\hline & 21 & 42 & \\
\hline \multicolumn{4}{|c|}{ C.A } \\
\hline D1 & $1,68^{A}$ & $1,56^{\mathrm{A}}$ & \multirow{3}{*}{0,02} \\
\hline D2 & $1,67^{\mathrm{A}}$ & $1,46^{\mathrm{AB}}$ & \\
\hline D3 & $1,57^{\mathrm{A}}$ & $1,41^{\mathrm{B}}$ & \\
\hline \multicolumn{4}{|c|}{ G.A.P } \\
\hline D1 & $595,5^{\mathrm{A}}$ & $2808,5^{A}$ & \multirow{3}{*}{2,31} \\
\hline D2 & $598^{A}$ & $2887,5^{\text {B }}$ & \\
\hline D3 & $628,6^{B}$ & $2950,5^{c}$ & \\
\hline \multicolumn{4}{|c|}{$\begin{array}{l}\text { A,B,C Comparaciones dentro de una misma columna, medias con diferente letra son } \\
\text { estadísticamente diferentes ( } \mathrm{P}<0,05) \text {. EEM: Error estándar de la media. D1: } \\
\text { Dieta control sin antibiótico ni probiótico. D2: Alimento comercial con antibiótico. } \\
\text { D3: Alimento comercial con Bacillus subtilis. C.A (conversión alimenticia), G.A.P } \\
\text { (ganancia acumulada de peso). }\end{array}$} \\
\hline
\end{tabular}


Es importante resaltar que la glucosa es la principal fuente para la obtención de energía necesaria para llevar a cabo los procesos metabólicos del organismo implicados en el anabolismo, por lo cual sus concentraciones en sangre son un factor importante para el buen funcionamiento de las vías metabólicas implicadas en la acreción proteica del músculo [28]. En el presente estudio, el uso de Bacillus subtilis incrementó las concentraciones de glucosa en sangre de manera significativa con respecto a los demás tratamientos, lo cual concuerda con los resultados encontrados en algunos estudios, en donde las aves alimentadas con una mezcla comercial de probióticos presentaron niveles mayores de glucosa en comparación con aquellas aves que no recibieron probióticos $[28,29]$. Sin embargo, algunos estudios realizados en pollos de engorde han mostrado que el uso de Bacillus subtilis en el alimento disminuye de manera significativa los niveles de glucosa en comparación con aquellas aves que recibieron una dieta sin adición de ningún agente antimicrobiano en el alimento [26,27].

Se ha encontrado que el uso de probióticos en la dieta favorece el desarrollo morfométrico del intestino, incrementando la altura y ancho de las vellosidades, lo cual se ve reflejado en una mayor área de contacto con el contenido intestinal, haciendo más eficiente los procesos de absorción de algunos nutrientes, principalmente calcio y fósforo, lo cual puede ser corroborado en los resultados obtenidos en el presente estudio, en el cual las aves que recibieron Bacillus subtilis en la dieta, presentaron concentraciones más altas de calcio y fósforo en sangre en comparación con los otros tratamientos [30,31,32]. Sin embargo, un estudio realizado por Saksrithai, Willits, \& King, 2019, en gallinas ponedoras, a las cuales se les suministró una mezcla de probióticos en el agua de bebida, encontraron que el uso de probióticos no tuvo un efecto significativo en la concentración de calcio y fósforo en sangre con respecto a aquellas aves que recibieron un APC en la dieta [33].

Se ha evidenciado que los probióticos actúan bajo diferentes modos de acción, mejorando el rendimiento productivo y la salud intestinal de las aves. Los probióticos pueden mejorar el rendimiento productivo de las aves manteniendo un balance saludable de bacterias en el intestino por exclusión competitiva contra las bacterias patógenas o inhibiendo su crecimiento por la producción de ácidos orgánicos, lo cual mejora el ambiente intestinal y favorece la absorción de nutrientes [7,10]. Algunos estudios realizados en aves han mostrado que el uso de Bacillus subtilis tienen efectos positivos sobre los parámetros productivos, incrementando las ganancias de peso y mejorando la tasa de conversión alimenticia $[34,35,36]$. Los incrementos en el rendimiento productivo son el resultado de una mayor eficiencia en los procesos de digestión y absorción de los nutrientes. Un trabajo de campo realizado por Jayaraman et al., (2017) reportaron que la inclusión de Bacillus subtilis mejora la histomorfología del intestino, principalmente incrementando la altura y ancho de las vellosidades a nivel de duodeno y yeyuno, segmentos donde se dan los mayores procesos de digestión y absorción de los nutrientes a nivel intestinal [37], mejorando así la digestibilidad de los nutrientes.

Boroojeni et al., (2018), reportaron que la suplementación de B. subtilis mejora la digestibilidad aparente, haciendo más eficiente el uso de los nutrientes presentes en la dieta, lo cual se ve reflejado en mayores ganancias diarias de peso y una menor tasa de conversión alimenticia [38].

\section{CONCLUSIONES}

La inclusión de Bacillus subtilis en la dieta de pollos de engorde constituye una alternativa para la sustitución parcial de antibióticos como promotores de crecimiento, ya que supone un incremento en metabolitos sanguíneos como glucosa, calcio y fósforo, y una disminución en el perfil lipídico, ALT y creatinina; además de mejorar el rendimiento productivo de las aves, indicando una mayor eficiencia en los procesos de absorción y aprovechamiento de los nutrientes suministrados en la dieta. 


\section{REFERENCIAS}

[1] GUZMAN, Y.E.; NAVARRO C.A.; ROA, M.L. Efecto del uso de probióticos en la morfometría intestinal de pollos de engorde. Archivos De Zootecnia, v. 67, n. 257, 2018, p. 93-98.

https://doi.org/10.21071/az.v0i0.3878

[2] FENELON, MARK; HAYES, MARIA; HENCHION, MAEVE; MULLEN, ANNE-MARIA; TIWARI, BRIJESH. Future protein supply and demand: strategies and factors Influencing a sustainable equilibrium. Foods, $v$. 6, n. 7, 2017, p. 1-21. $10.3390 /$ foods 6070053

[3] SOKALE, A.O.; MENCONI, A.; MATHIS, G.F.; LUMPKINS, B.; SIMS, M.D.; WHELAN, R.A.; DORANALLI, K. Effect of Bacillus subtilis DSM 32315 on the intestinal structural integrity and growth performance of broiler chickens under necrotic enteritis challenge. Poultry Science, v. 98, n. 11, 2019, p. 5392-5400. https://doi.org/10.3382/ps/pez368

[4] KROCKO, MIROSLAV; CANIGOVÁ, MARGITA; BEZEKOVA, JANA; LAVOVÁ, MONIKA; HASCÍK, PETER; DUCKOVÁ, VIERA. Effect of nutrition with propolis and bee pollen supplements on bacteria colonization pattern in gastrointestinal tract of broiler chickens. Scientific Papers Animal Science and Biotechnologies, v. 45, n. 1, 2012, p. 63-67.

[5] KIM, IN-HO; MOHAMMADIGHEISAR, MOHSEN. Phytobiotics in poultry and swine nutrition - a review. Italian Journal Of Animal Science, v. 17, n. 1, 2018, p. 92-99. 10.1080/1828051X.2017.1350120

[6] REIS, M.P.; FASSANI, E.J.; GARCIA-JÚNIOR, A.A.P.; RODRIGUES, P.B.; BERTECHINI, A.G.; BARRET, N.; PERSIA, M.E.; SCHMIDT, C.J. Effect of Bacillus subtilis (DSM 17299) on performance, digestibility, intestine morphology, and pH in broiler chickens. Journal Of Applied Poultry Research, v. 26, n. 4, 2017, p. 573-583. https://doi.org/10.3382/japr/pfx032

[7] SUGIHARTO, SUGIHARTO. Role of nutraceuticals in gut health and growth performance of poultry. Journal Of The Saudi Society Of Agricultural Sciences, v. 15, n. 2, 2016, p. 99-111. https://doi.org/10.1016/j.jssas.2014.06.001

[8] MEHDI,YOUCEF; LÉTOURNEAU-MONTMINT, MARIE-PIERRE; GAUCHER, MARIE-LOU; CHORFI,YOUNES; SURESH, GAYATRI; ROUISSI, TAREK; KAUR-BRAR, SATINDER; COTÉ, CAROLINE; AVALOS-RAMIREZ, ANTONIO; GODBOUT, STÉPHANE. Use of antibiotics in broiler production: global impacts and alternatives. Animal Nutrition, v. 4, n. 2, 2018, p. 170-178.

https://doi.org/10.1016/j.aninu.2018.03.002

[9] SALIM, HOSSAN M.D.; SHAHIDUL-HUQUE, KHAN; KAMARUDDIN, KAZI M.; HAQUE-BEG, ANWARUL. Global restriction of using antibiotic growth promoters and alternative strategies in poultry production. Science Progress, v. 101, n. 1, 2018, p. 52-75. 10.3184/003685018X15173975498947

[10] NAGAE-KURITZA, LEANDRO; WESTPHAL, PATRICK; SANTIN, ELIZABETH. Probióticos na avicultura. Ciência Rural, v. 44. n. 8, 2014, p. 1457-1465. 10.1590/0103-8478cr20120220

[11] BROWN, KIRSTY; UWIERA, RICHAR, R.E.; KALMOKOFF, MARTIN L.; BROOKS, STEVE P.J.; INGLIS, DOUGLAS. Antimicrobial growth promoter use in livestock: a requirement to understand their modes of action to develop effective alternatives. International Journal Of Antimicrobial Agents, v. 49, n. 1, 2017, p. 12-24. https://doi.org/10.1016/j.ijantimicag.2016.08.006

[12] QIN, CHUBIN; GONG, LI; ZHANG, XIAOPING; WANG, YUANYUAN; WANG, YIBIN; WANG, BAIKUI; LI, YALI; LI, WEIFEN. Effect of Saccharomyces boulardii and Bacillus subtilis B10 on gut microbiota modulation in broilers. Animal Nutrition, v. 55, n. 3, 2018, p. 63-67.

https://doi.org/10.1016/j.aninu.2018.03.004

[13] LONDOÑO-PÉREZ, SANTIAGO; PARRA-SUESCÚN, JAIME. Efecto de la adición de cepas probióticas sobre metabolitos sanguíneos en cerdos en crecimiento. Biotecnología en el Sector Agropecuario y Agroindustrial, v. 13, n. 2, 2015, p. 45-56.

10.18684/BSAA(13)49-56 
[14] AVIAGEN. Ross 308 AP. Objetivo de rendimiento. 2017. Disponible: http://es.aviagen.com/assets/Tech_Center/BB_Foreign_Language_Docs/Spanish_TechDocs/Ross308AP-Broiler-PO-2017-ES.pdf [citado 22 de Julio de 2020].

[15] LÓPEZ-HERRERA, A.; MADRID-GARCÉS, T.A.; PARRA-SUESCÚN, J.E. La ingesta de aceite esencial de orégano (Lippia origanoides) mejora la morfología intestinal en Broilers. Archivos De Zootecnia, v. 66, n. 254, 2017, p. 287-299. https://doi.org/10.21071/az.v0i0.3876

[16] CHÁVEZ, L.A.; LÓPEZ, A.; PARRA, J.E. Crecimiento y desarrollo intestinal de aves de engorde alimentadas con cepas probióticas. Archivos De Zootecnia, v. 65, n. 249, 2016, p. 51-58. https://doi.org/10.21071/az.v65i249.441

[17] SAS/STAT®. Institute Inc. Statistical Analysis Systems Institute. User's Guide, Version 9.4th Ed. Cary, NC: SAS Institute Inc. 2017. Disponible: https://www.sas.com/content/dam/SAS/en_us/doc/factsheet/stat-101372. pdf [citado 22 de Julio de 2020].

[18] AHMAD, NAZIM; HAQUE, IQRAMUL; ALAM-MIAH, MOHAMMAD. Comparative analysis of body weight and serum biochemistry in broilers supplemented with some selected probiotics and antibiotic growth promoters. Journal Of Advanced Veterinary And Animal Research, v. 4, n. 3, 2017, p. 288-294. http://doi.org/10.5455/javar.2017.d226

[19] ABRAMOWICZ, KATARZYNA; KRAUZE, MAGDALENA; OGNIK, KATARZYNA. The effect of a probiotic preparation containing Bacillus subtilis PB6 in the diet of chickens on redox and biochemical parameters in their blood. Annals Of Animal Science, 2019, p. 1-33. 10.2478/aoas-2018-0059

[20] HEDAYATI, M.; KHALAJI, S.; MANAFI, M.; PIRANY, N. Efficacy of Bacillus subtilis and bacitracin methylene disalicylate on growth performance, digestibility, blood metabolites, immunity, and intestinal microbiota after intramuscular inoculation with Escherichia coli in broilers. Poultry Science, v. 95, n. 5, 2017, p. 1174-1183. https://doi.org/10.3382/ps/pew347

[21] CALVO, E., CELI, PIETRO; KLUENTER, A.M., PÉREZ- SCHMEISSER, J.; VERLHAC, VIVIANE. Biomarkers of gastrointestinal functionality in animal nutrition and health. Animal Feed Science And Technology, v. 250, n. 1, 2019, p. 9-31. https://doi.org/10.1016/j.anifeedsci.2018.07.012

[22] JAKHAR, K.K.; KUMAR, SARVAN; NEHRA, VIKAS; SHARMA, VIKASH. Biochemical studies in experimentally Escherichia coli infected broiler chicken supplemented with neem (Azadirachta indica) leaf extract. Veterinary World, v. 8, n. 11, 2015, p. 1340-1345. 10.14202/vetworld.2015.1340-1345

[23] ISMAIL, HAGER-TAREK. Biochemical and hematological studies on the effect of Neem (Azadirachta indica) leaves aqueous extract on Newcastle disease vaccine and infection in broiler chickens. International Journal Of Recent Scientific Research, v. 8, n. 3, 2017, p. 15876-15884. http://dx.doi.org/10.24327/ijrsr.2017.0803.0002

[24] HUSSEIN, E.; SELIM, S. Efficacy of yeast and multi-strain probiotic alone or in combination on growth performance, carcass traits, blood biochemical constituents, and meat quality of broiler chickens. Livestock Science, v. 216, n. 1, 2018, p. 153-159. https://doi.org/10.1016/j.livsci.2018.08.008

[25] FOO, H.L.; HASHEMI, S.M.; LOH, T.C. Liquid metabolite of Lactobacillus plantarum and putrescine effects on growth, tissue polyamine, blood lipids and intestine morphology of broiler chickens. Iranian Journal Of Applied Animal Science, v. 8, n. 2, 2018, p. 333-342.

[26] GONG, LI; WANG, BAIKUI; MEI, XIAOQIANG; XU, HAN; QIN, YAN; LI, WEIFEN; ZHOU, YINGSHAN. Effects of three probiotic Bacillus on growth performance, digestive enzyme activities, antioxidative capacity, serum immunity, and biochemical parameters in broilers. Animal Science Journal, v. 89, n. 11, 2018, p. 1561-1571.

https://doi.org/10.1111/asj.13089 
[27] AL-BAADANI, HANI H.; ABUDABOS, ALAELDEIN; AL-MUFARREJ, SAUD; AL-ALI A.; ALHIDARY, IBRAHIM. Dietary supplementation of Bacillus subtilis, Saccharomyces cerevisiae and their symbiotic effect on serum biochemical parameters in broilers challenged with Clostridium perfringens. Journal Of Applied Animal Research, v. 46, n. 1, 2018, p.1064-1072. https://doi.org/10.1080/09712119.2018.1454325

[28] ABBAS, GHULAM; ASIF-IQBAL, MUHAMMAD; RIAZ, MAHAM; SAJID, MUHAMMAD; ZAHID, OSAMA; WASIM-ABBAS, SYED; SAEED, HIRA; IMRAN-RAZA, ALI; ZOHAIB, MALIK. Comparative effect of different levels of probiotics (protexin) on hemato-chemical profile in broilers. Advances In Zoology And Botany, v. 6, n. 3, 2018, p. 84-87. 10.13189/azb.2018.060302

[29] CORAZZIN, MIRCO; POURNAZARI, MARYAM; AA-QOTBI, ALI; SEIDAVI, ALIREZA. Prebiotics, probiotics and thyme (Thymus vulgaris) for broilers: performance, carcass traits and blood variables. Revista Colombiana De Ciencias Pecuarias, v. 30, n. 1, 2017, p. 3-10. 10.17533/udea.rccp.v30n1a01

[30] XING, Y.; WANG, S.; FAN, J.; OSO, A.O.; KIM, S.W.; XIAO, D.; YANG, T.; LIU, G.; JIANG, G.; LI, Z.; LI, L.; ZHANG, B. Effects of dietary supplementation with lysine-yielding Bacillus subtilis on gut morphology, cecal microflora, and intestinal immune response of Linwu ducks. Journal Of Animal Science, v. 93, n. 7, 2015, p. 3449-3457. 10.2527/jas.2014-8090

[31] CHEN, YUEPING; WEN, CHAO; ZHOU, YANMIN. Dietary synbiotic incorporation as an alternative to antibiotic improves growth performance, intestinal morphology, immunity and antioxidant capacity of broilers. Journal Of The Science Of Food And Agriculture, v. 98, n. 9, 2018, p. 3343-3350. https://doi.org/10.1002/jsfa.8838

[32] GAO, Y.P.; MIN, Y.N.; YANG, H.L.; XU, Y.X. Effects of dietary supplementation of synbiotics on growth performance, intestinal morphology, slgA content and antioxidant capacities of broilers. Journal Of Animal Physiology And Animal Nutrition, v. 100, n. 6, 2016, p. 1073-1080. 10.1111/jpn.12479

[33] KING, A.J.; SAKSRITHAI, KETWEE; WILLITS, N.H. Production performance of laying hens at peak lay, sulfur compounds in manure, and selected serum profiles: efficacy of Lactobacillus species as probiotics. Animal Production Science, v. 60, n. 2, 2019, p. 296-304. 10.1071/AN18724

[34] BRINCH, K.S.; GERAERT, P.A.; JACQUIER, V.; NELSON, A.; NIELSEN, P.; RHAYAT, L.; DEVILLARD, E. Bacillus subtilis strain specificity affects performance improvement in broilers. Poultry Science, v. 96, n. 7, 2017, p. 2274-2280. https://doi.org/10.3382/ps/pex018

[35] KIM, I.H.; RUDEAUX, F.; UPADHAYA, S.D. Effects of inclusion of Bacillus subtilis (Gallipro) to energy- and protein-reduced diet on growth performance, nutrient digestibility, and meat quality and gas emission in broilers. Poultry Science, v. 98, n. 5, 2019, p. 2169-2178. https://doi.org/10.3382/ps/pey573

[36] KIM, I.H.; PARK, J.H.; YUN, H.M. The effect of dietary Bacillus subtilis supplementation on the growth performance, blood profile, nutrient retention, and caecal microflora in broiler chickens. Journal Of Applied Animal Research, v. 46, n.1, 2018, p. 868-872.

https://www.tandfonline.com/loi/taar20

[37] CHATTERJEE, PARESH-NATH; DAS, PARTHA-PRATIM; JAYARAMAN, SATHISHKUMAR; ROY, BARUN; SAINI, PRAKASH-CHANDRA. Use of Bacillus Subtilis PB6 as a potential antibiotic growth promoter replacement in improving performance of broiler birds. Poultry Science, v. 96, n. 8, 2017, p. 2614-2622. https://doi.org/10.3382/ps/pex079

[38] GOODARZI-BOROOJENI, FARSHAD; VAHJEN, W.; MANNER, K.; BLANCH, A.; SANDVANG, D.; ZENTEK, J. Bacillus subtilis in broiler diets with different levels of energy and protein. Poultry Science, v. 97, n. 11, 2018, p. 3967-3976. 10.3382/ps/pey265

[39] ANTHONY, R. et al. Guidelines for the Euthanasia of Animals. 5 ed. Illinois (United States): American Veterinary Medical Association, 2013, $121 \mathrm{p}$. 